\title{
Refractory Kidney Medullary Carcinoma
}

National Cancer Institute

\section{Source}

National Cancer Institute. Refractory Kidney Medullary Carcinoma. NCI Thesaurus. Code C162727.

A kidney medullary carcinoma that does not respond to treatment. 